\title{
A FAMILY OF STRONGLY SINGULAR OPERATORS
}

\author{
MAGALI FOLCH-GABAYET
}

(Received 28 August 1998; revised 25 March 1999)

Communicated by A. H. Dooley

\begin{abstract}
Let $\psi$ be a positive function defined near the origin such that $\lim _{t \rightarrow 0^{+}} \psi(t)=0$. We consider the operator $T_{z} f$, defined as the principal value of the convolution of a function $f$ and a kernel $K(t)=e^{i \gamma(t)} t^{-z} / \psi(t)^{1-z}$, where $z$ is a complex number, $0 \leq \operatorname{Re}(z) \leq 1,0<t \leq 1$ and $\gamma$ is a real function. Assuming certain regularity conditions on $\psi$ and $\gamma$ and certain relations between $\psi$ and $\gamma$ we show that $T_{\theta}$ is a bounded operator on $L^{p}(\mathbb{R})$ for $1 / p=(1+\theta) / 2$ and $0 \leq \theta<1$, and $T_{1}$ is bounded from $H^{1}(\mathbb{R})$ to $L^{1}(\mathbb{R})$.
\end{abstract}

1991 Mathematics subject classification (Amer. Math. Soc.): primary 42A45, 42A85, 43A32, 44A05, $44 \mathrm{~A} 35$.

\section{Introduction}

Consider the following operator, defined for functions in $C_{0}^{\infty}(\mathbb{R})$

$$
T_{\alpha \beta} f(x)=\lim _{\epsilon \rightarrow 0^{+}} \int_{\epsilon}^{1} e^{i t^{-\alpha}} f(x-t) \frac{d t}{t^{\beta}},
$$

where $\alpha>0$ and $\beta>1$. Hirschman studied this operator and proved the following theorem in [2].

THEOREM 1.1. Let $\alpha>0$ and $\beta>1$. Whenever $\alpha+2 \geq 2 \beta$ the following holds:

(i) $T_{\alpha \beta}$ extends to a bounded operator on $L^{2}(\mathbb{R})$.

(ii) If $|1 / 2-1 / p|<1 / 2-(\beta-1) / \alpha$ then $T_{\alpha \beta}$ extends to a bounded operator on $L^{p}(\mathbb{R})$.

(C) 1999 Australian Mathematical Society $0263-6115 / 99 \$ A 2.00+0.00$ 
(iii) If $|1 / 2-1 / p|>1 / 2-(\beta-1) / \alpha$ then $T_{\alpha \beta}$ is not a bounded operator on $L^{p}(\mathbb{R})$.

Fefferman and Stein considered the case $|1 / 2-1 / p|=1 / 2-(\beta-1) / \alpha$ in [1]. Their results complete Theorem 1.1.

THEOREM 1.2. Let $\alpha>0$ and $\beta>1$ such that $\alpha+2 \geq 2 \beta$. If

$$
\left|\frac{1}{2}-\frac{1}{p}\right| \leq \frac{1}{2}-\frac{\beta-1}{\alpha}
$$

then $T_{\alpha \beta}$ extends to a bounded operator on $L^{p}(\mathbb{R})$, for $1<p<\infty$.

In the present work we are interested in studying the operator when the singularity at zero is worse than a power. An example would be

$$
T f(x)=\lim _{\epsilon \rightarrow 0^{+}} \int_{\epsilon}^{l} e^{i \gamma(t)} f(x-t) \frac{e^{1 / t}}{t} d t,
$$

where $\gamma$ is a real-valued function.

To compensate for the singularity at the origin, the phase function $\gamma$ should approach infinity fast as the argument tends to zero. For $f$ in $C_{0}^{\infty}(\mathbb{R})$ we consider the operator

$$
T f(x)=\lim _{\epsilon \rightarrow 0^{+}} \int_{\epsilon}^{1} e^{i \gamma(t)} f(x-t) \frac{d t}{\Phi(t)},
$$

where $\lim _{t \rightarrow 0^{+}} \Phi(t)=0$ and $\lim _{t \rightarrow 0^{+}} \Phi^{\prime}(t)=0$.

The first thing to do is to understand under what conditions $T$ is a bounded operator on $L^{2}(\mathbb{R})$.

When $\Phi$ approaches zero faster than a power, $\gamma$ has to approach infinity faster than the reciprocal of a power. It is clear that when $\Phi$ is supported in the interval $[0,1]$, the behavior of $\gamma$ near zero should be dictated by that of $\Phi$.

In the example given by equation (1) it turns out that if $\gamma$ is such that $\gamma^{\prime}(t)=e^{2 / t}$, for example $\gamma(t)=-\int_{t}^{1} e^{2 / s} d s$, then $T$ is bounded on $L^{2}(\mathbb{R})$.

If we now choose $\gamma$ so that $\gamma^{\prime \prime}(t)=t^{2 b-2} e^{2 / t}$, for $b>0, T$ will not be bounded on $L^{2}(\mathbb{R})$.

For this choice of $\Phi$ and $\gamma$ we have that

$$
\lim _{t \rightarrow 0^{+}} \frac{1}{|\Phi(t)| \sqrt{\left|\gamma^{\prime \prime}(t)\right|}}=\lim _{t \rightarrow 0^{+}} t^{-b}=\infty .
$$

Assuming some regularity conditions on $\gamma$ and $\Phi$ we will show that if

$$
\left|\frac{1}{\Phi(t)}\right| \leq C \sqrt{\left|\gamma^{\prime \prime}(t)\right|}
$$


then $T$ is bounded on $L^{2}(\mathbb{B})$.

On the other hand if

$$
\lim _{t \rightarrow 0^{+}} \frac{1}{|\Phi(t)| \sqrt{\left|\gamma^{\prime \prime}(t)\right|}}=\infty
$$

then $T$ will not be bounded on $L^{2}(\mathbb{R})$.

Notice that when $\Phi(t)=t^{\beta}$ and $\gamma(t)=t^{-\alpha}$ the above statements imply that $T_{\alpha \beta}$ is bounded on $L^{2}(\mathbb{R})$ only when $\alpha / 2+1-\beta \geq 0$, which is Hirschman's result for $p=2$.

We now turn our attention to the study of $T$ on $L^{1}(\mathbb{R})$. The only way $T$ can be bounded on $L^{1}(\mathbb{R})$ is when the function $1 / \Phi$ is integrable near zero. Since $1 / \Phi(t)=t^{-1}$ just fails to be integrable near zero, there is some hope that

$$
T f(x)=\lim _{\epsilon \rightarrow 0^{+}} \int_{\epsilon}^{1} e^{i \gamma(t)} f(x-t) \frac{d t}{t},
$$

is a bounded operator from $H^{1}(\mathbb{R})$ to $L^{1}(\mathbb{R})$, due to the oscillatory factor $e^{i \gamma(t)}$.

Fefferman and Stein proved this statement when $\gamma$ is the reciprocal of a power (see [1]). Theorem 1.2 follows as a corollary.

When $\gamma$ approaches zero faster than the reciprocal of a power we will also have that $T$, as defined in (5), is a bounded operator from $H^{1}(\mathbb{R})$ to $L^{1}(\mathbb{R})$.

The above discussion leads us to consider a family of operators

$$
T_{z} f(x)=\lim _{\epsilon \rightarrow 0^{+}} \int_{\epsilon}^{1} e^{i \gamma(t)} f(x-t) \frac{t^{-z}}{\psi(t)^{1-z}} d t,
$$

where $z=a+i b, 0 \leq a \leq 1$ and $\psi$ is a positive function that satisfies

$$
\frac{1}{\psi(t)} \leq C \sqrt{\left|\gamma^{\prime \prime}(t)\right|}
$$

In this setting one of our tasks is to prove that $T_{0}$ is a bounded operator on $L^{2}(\mathbb{R})$ and $T_{1}$ is a bounded operator from $H^{1}(\mathbb{R})$ to $L^{1}(\mathbb{R})$. To do so we will impose the following regularity conditions on $\gamma$ and $\psi$.

1.1. Assumptions and results (a.1) We will assume that $\gamma$ and $\gamma^{\prime}$ are monotone, guaranteeing the existence of the inverse of $\gamma^{\prime}$, denoted $\gamma^{\prime-1}$. Without loss of generality we will take $\gamma^{\prime}(t)>0$, with $\gamma^{\prime}$ decreasing on $(0,1]$.

(a.2) As discussed before, for the operator to be bounded on $L^{2}(\mathbb{R})$ we will need to assume $1 / \psi(t) \leq C \sqrt{\left|\gamma^{\prime \prime}(t)\right|}$, for $t>0$ close to zero and $C$ a constant. However to prove that the operator in bounded from $H^{1}(\mathbb{R})$ to $L^{1}(\mathbb{R})$ we will assume the stronger condition

$$
\left|\frac{\psi^{\prime}(t)}{\psi(t)}\right| \leq \frac{1}{2}\left|\frac{\gamma^{\prime \prime \prime}(t)}{\gamma^{\prime \prime}(t)}\right|
$$


for $t>0$ close to zero and $C$ a constant. We will take $\psi \in C^{2}[0,1]$ and $\gamma \in C^{3}(0,1]$.

The fact that (7) implies that $1 / \psi(t) \leq C \sqrt{\left|\gamma^{\prime \prime}(t)\right|}$ will be proved below.

(a.3) Several growth conditions on $\gamma^{\prime \prime}$ and $\gamma^{\prime}$ will be assumed. One of these is that $\gamma^{\prime \prime}$ is to be roughly constant where $\gamma^{\prime}$ 'doubles'. We write that as follows:

$$
\left|\gamma^{\prime \prime}\left(\gamma^{\prime-1}(2 s)\right)\right| \leq C\left|\gamma^{\prime \prime}\left(\gamma^{\prime-1}(s)\right)\right|
$$

where $C$ is a constant bigger than one independent of $s$.

(a.4) We will assume that there exists a constant $A$ and $\epsilon>0$ with $A>1+\epsilon$ such that

$$
\gamma^{\prime}(t) \geq A \gamma^{\prime}((1+\epsilon) t)
$$

for $t>0$ close to zero.

(a.5) The last condition on the growth of $\gamma$ is the following. There exists a $\lambda$ such that $1 / 2<\lambda<1$, and a constant $C$ such that

$$
\left|\gamma^{\prime \prime}(t)\right| \leq C \gamma^{\prime}(t)^{2 \lambda},
$$

for $t>0$ close to zero.

(a.6) Finally we will assume that $\psi, \psi^{\prime}$ and $\gamma^{\prime \prime}$ are monotone.

Unless otherwise noted, we will assume throughout that $\psi$ and $\gamma$ satisfy assumptions (a.1) through (a.6) in Theorem 1.3 through Theorem 1.5 below.

Examples: $\gamma^{\prime}(t)=e^{1 / 2}, e^{e^{\prime \prime \prime}}, t^{-\alpha}, e^{(\ln (1 / t))^{\alpha}}$ for $\alpha>1$ satisfy (a.1) through (a.6).

Let us prove now that inequality (7) implies that $1 / \psi(t) \leq C \sqrt{\left|\gamma^{\prime \prime}(t)\right|}$. For $0<t<1$ integrate both sides of (7) from $t$ to 1 .

$$
\int_{t}^{1}\left|\frac{\psi^{\prime}(s)}{\psi(s)}\right| d s \leq \frac{1}{2} \int_{t}^{1}\left|\frac{\gamma^{\prime \prime \prime}(s)}{\gamma^{\prime \prime}(s)}\right| d s .
$$

Since $\psi$ and $\gamma^{\prime \prime}$ are monotone we get

$$
\ln \left(\frac{\psi(1)}{\psi(t)}\right) \leq \frac{1}{2} \ln \left(\frac{\left|\gamma^{\prime \prime}(t)\right|}{\left|\gamma^{\prime \prime}(1)\right|}\right)
$$

and so $1 / \psi(t) \leq C \sqrt{\left|\gamma^{\prime \prime}(t)\right|}$.

Assuming that the limit in (6) exists we now state the results on $T_{z}$. If $z=\theta$, for $0 \leq \theta \leq 1$, we have

$$
T_{\theta} f(x)=\lim _{\epsilon \rightarrow 0^{+}} \int_{\epsilon}^{1} e^{i v(t)} f(x-t) \frac{t^{-\theta}}{\psi(t)^{1-\theta}} d t .
$$

The main theorem for $T_{\theta}$ is the following. 
THEOREM 1.3. (i) $T_{\theta}$ is a bounded operator on $L^{p}(\mathbb{R})$ for $1 / p=(1+\theta) / 2$ and $0 \leq \theta<1$, with $\left\|T_{\theta}\right\|_{L^{p} \rightarrow L^{p}} \leq A_{\theta}$ where $A_{\theta}$ depends only on $\theta$.

(ii) $T_{1}$ is a bounded operator from $H^{1}(\mathbb{R})$ to $L^{1}(\mathbb{R})$.

Theorem 1.3 will be obtained from the two following results.

THEOREM 1.4. $T_{i b}$ extends to a bounded operator on $L^{2}(\mathbb{R})$ with $\left\|T_{i b}\right\|_{L^{2} \rightarrow L^{2}} \leq A_{b}$, where $A_{b}=O(|b|+1)$.

THEOREM 1.5. $T_{1+i b}$ is a bounded operator from $H^{1}(\mathbb{R})$ to $L^{1}(\mathbb{R})$ with $\left\|T_{1+i b}\right\|_{H^{1} \rightarrow L^{1}}$ $\leq A_{b}$, where $A_{b}=O(|b|+1)$.

Theorem 1.1 says that $T_{\alpha \beta}$ will not be bounded on $L^{2}(\mathbb{R})$ when $2+\alpha<2 \beta$. This result is generalized as follows.

THEOREM 1.6. Suppose that $\gamma, \gamma^{\prime}, \gamma^{\prime \prime}$ and $\psi$ are monotone and that

$$
\lim _{\epsilon \rightarrow 0^{+}} \frac{\gamma^{\prime \prime}(\epsilon)}{\gamma^{\prime 2}(\epsilon)}=0
$$

Also suppose there is a constant $A>1$ such that

$$
\gamma^{\prime}\left(|\gamma|^{-1}(2 s)\right) \leq A \gamma^{\prime}\left(|\gamma|^{-1}(s)\right)
$$

for all large $s>0$. Then if $\lim _{t \rightarrow 0^{+}} 1 /\left(|\psi(t)| \sqrt{\left|\gamma^{\prime \prime}(t)\right|}\right)=\infty, T_{0}$ is not bounded on $L^{2}(\mathbb{R})$.

In what follows $C$ will denote a constant that may change from line to line.

1.2. Existence Let $f$ be a function in $C_{0}^{\infty}(\mathbb{R})$. To see that under assumptions (a.1) through (a.6) the limit in (6) exists, we integrate by parts. For $0<\epsilon^{\prime} \leq \epsilon$ we write

$$
\begin{aligned}
& \int_{\epsilon^{\prime}}^{\epsilon} e^{i \gamma(t)} f(x-t) \frac{t^{-z}}{\psi(t)^{1-z}} d t=\frac{1}{i} \int_{\epsilon^{\prime}}^{\epsilon} \frac{d}{d t}\left(e^{i \gamma(t)}\right) \frac{f(x-t)}{\gamma^{\prime}(t)} \frac{t^{-z}}{\psi(t)^{1-z}} d t \\
& \quad=\left.\frac{1}{i} e^{i \gamma(t)} \frac{f(x-t)}{\gamma^{\prime}(t)} \frac{t^{-z}}{\psi(t)^{1-z}}\right|_{\epsilon^{\prime}} ^{\epsilon}-\frac{1}{i} \int_{\epsilon^{\prime}}^{\epsilon} e^{i \gamma(t)} \frac{d}{d t}\left(\frac{(f(x-t)}{\gamma^{\prime}(t)} \frac{t^{-z}}{\psi(t)^{1-z}}\right) d t \\
& =\mathrm{I}_{\epsilon}+\mathrm{II}_{\epsilon} .
\end{aligned}
$$

Let $z=a+i b$ with $0 \leq a \leq 1$. Then

$$
\left|\mathrm{I}_{\epsilon}\right| \leq \frac{\left|f\left(x-\epsilon^{\prime}\right)\right| \epsilon^{\prime-a}}{\gamma^{\prime}\left(\epsilon^{\prime}\right) \psi\left(\epsilon^{\prime}\right)^{1-a}}+\frac{|f(x-\epsilon)| \epsilon^{-a}}{\gamma^{\prime}(\epsilon) \psi(\epsilon)^{1-a}} .
$$


Since $\lim _{t \rightarrow 0^{+}} \psi^{\prime}(t)=0$, we have that for $t$ close to zero, $\psi(t) \leq t$. Using this and the fact that $1 / \psi(t) \leq C \sqrt{\left|\gamma^{\prime \prime}(t)\right|}$ we have that

$$
\frac{\epsilon^{-a}}{\gamma^{\prime}(\epsilon) \psi(\epsilon)^{1-a}} \leq C \frac{\sqrt{\left|\gamma^{\prime \prime}(\epsilon)\right|}}{\gamma^{\prime}(\epsilon)} \epsilon^{-a} \psi(\epsilon)^{a} \leq C \frac{\sqrt{\left|\gamma^{\prime \prime}(\epsilon)\right|}}{\gamma^{\prime}(\epsilon)} .
$$

Assumption (a.5) implies that $\lim _{\epsilon \rightarrow 0^{+}} \sqrt{\left|\gamma^{\prime \prime}(\epsilon)\right|} / \gamma^{\prime}(\epsilon)=0$. Hence

$$
\lim _{\epsilon \rightarrow 0^{+}}\left|I_{\epsilon}\right|=0
$$

Let's now estimate $\mathrm{II}_{\epsilon}$.

$$
\begin{aligned}
\left|\mathrm{II}_{\epsilon}\right| \leq & \int_{\epsilon^{\prime}}^{\epsilon} \frac{\left|f^{\prime}(x-t)\right| t^{-a}}{\gamma^{\prime}(t) \psi(t)^{1-a}} d t+\int_{\epsilon^{\prime}}^{\epsilon}|z| \frac{|f(x-t)| t^{-a-1}}{\gamma^{\prime}(t) \psi(t)^{1-a}} d t \\
& +\int_{\epsilon^{\prime}}^{\epsilon} \frac{|f(x-t)| t^{-a}\left|\gamma^{\prime \prime}(t)\right|}{\gamma^{\prime 2}(t) \psi(t)^{1-a}} d t+\int_{\epsilon^{\prime}}^{\epsilon}|z-1| \frac{|f(x-t)| t^{-a}\left|\psi^{\prime}(t)\right|}{\gamma^{\prime}(t) \psi(t)^{2-a}} d t \\
= & \mathrm{II}_{\epsilon 1}+\mathrm{II}_{\epsilon 2}+\mathrm{II}_{\epsilon 3}+\mathrm{II}_{\epsilon 4} .
\end{aligned}
$$

As before we have that $\lim _{\epsilon \rightarrow 0^{+}} \epsilon^{-a} /\left(\gamma^{\prime}(\epsilon) \psi(\epsilon)^{1-a}\right)=0$ and hence

$$
\lim _{\epsilon \rightarrow 0^{+}}\left|I_{\epsilon 1}\right|=0
$$

Since $1 / \psi(t) \leq C \sqrt{\left|\gamma^{\prime \prime}(t)\right|}$ we see that

$$
\begin{aligned}
\left|\mathrm{II}_{\epsilon 2}\right| & \leq \max _{0 \leq t \leq 1}|f(x-t)||z| \int_{\epsilon^{\prime}}^{\epsilon} \frac{t^{-a-1}}{\gamma^{\prime}(t) \psi(t)^{1-a}} d t \\
& \leq C \max _{0 \leq t \leq 1}|f(x-t)||z| \int_{\epsilon^{\prime}}^{\epsilon} \frac{\sqrt{\left|\gamma^{\prime \prime}(t)\right|} t^{-a-1} \psi(t)^{a}}{\gamma^{\prime}(t)} d t .
\end{aligned}
$$

Since $\lim _{t \rightarrow 0^{+}} \gamma^{\prime \prime}(t) / \gamma^{\prime}(t)^{2}=0$, we have that $1 / \gamma^{\prime}(t) \leq C t$, for $t$ small. Using this together with assumption (a.5), $\sqrt{\left|\gamma^{\prime \prime}(t)\right|} \leq C \gamma^{\prime \lambda}(t)$ for some $1 / 2<\lambda<1$, and $\psi(t) \leq t$ we see that

$$
\begin{aligned}
\left|\mathrm{II}_{\epsilon 2}\right| & \leq C \max _{0 \leq t \leq 1}|f(x-t) \| z| \int_{\epsilon^{\prime}}^{\epsilon} \gamma^{\prime}(t)^{\lambda-1} t^{-1} d t \\
& \leq C \max _{0 \leq t \leq 1}|f(x-t)||z| \int_{\epsilon^{\prime}}^{\epsilon} t^{-\lambda} d t .
\end{aligned}
$$

Hence

$$
\lim _{\epsilon \rightarrow 0^{+}}\left|I_{\epsilon 2}\right|=0
$$


In a similar way we have

$$
\left|\mathbf{I I}_{\epsilon 3}\right| \leq C \max _{0 \leq t \leq 1}|f(x-t)| \int_{\epsilon^{\prime}}^{\epsilon} \frac{\left|\gamma^{\prime \prime}(t)\right|^{3 / 2}}{\gamma^{\prime 2}(t)} d t
$$

If $k^{\prime}$ is such that $\gamma^{\prime-1}\left(2^{k^{\prime}+1}\right)<\epsilon \leq \gamma^{\prime-1}\left(2^{k^{\prime}}\right)$ then we see that

$$
\begin{aligned}
\left|\mathrm{II}_{\epsilon 3}\right| & \leq C \max _{0 \leq t \leq 1}|f(x-t)| \sum_{k=k^{\prime}}^{\infty} \int_{\gamma^{\prime-1}\left(2^{k+1}\right)}^{\gamma^{\prime-1}\left(2^{k}\right)} \frac{\left|\gamma^{\prime \prime}(t)\right|}{\gamma^{\prime}(t)} \frac{\sqrt{\left|\gamma^{\prime \prime}(t)\right|}}{\gamma^{\prime}(t)} d t \\
& \leq C \max _{0 \leq t \leq 1}|f(x-t)| \sum_{k=k^{\prime}}^{\infty} \int_{\gamma^{\prime-1}\left(2^{k+1}\right)}^{\gamma^{\prime-1}\left(2^{k}\right)} \frac{\left|\gamma^{\prime \prime}(t)\right|}{\gamma^{\prime}(t)} \gamma^{\prime}(t)^{\lambda-1} d t \\
& \leq C \max _{0 \leq t \leq 1}|f(x-t)| \sum_{k=k^{\prime}}^{\infty}\left(2^{k}\right)^{\lambda-1} \ln (2) .
\end{aligned}
$$

As $\epsilon \rightarrow 0^{+}, k^{\prime} \rightarrow \infty$ and $\lambda-1<0$ hence we can conclude that

$$
\lim _{\epsilon \rightarrow 0^{+}}\left|\mathrm{II}_{\epsilon 3}\right|=0
$$

To bound $\mathrm{II}_{\epsilon 4}$ just notice that

$$
\left|\mathrm{II}_{\epsilon 4}\right| \leq C \max _{0 \leq t \leq 1}|f(x-t)||z-1| \int_{\epsilon^{\prime}}^{\epsilon} \frac{1}{\gamma^{\prime}(t)} \frac{\left|\psi^{\prime}(t)\right|}{\psi^{2}(t)} d t
$$

After an integration by parts, methods used before show that

$$
\lim _{\epsilon \rightarrow 0^{+}}\left|\mathrm{II}_{\epsilon 3}\right|=0
$$

This shows that the limit in (6) exists.

\section{Preliminaries}

Let $0<\epsilon<1$, and define

$$
T_{\epsilon, z} f(x)=\int_{\epsilon}^{l} e^{i \gamma(t)} f(x-t) \frac{t^{-z}}{\psi(t)^{1-z}} d t
$$

so that for $f$ in $C_{0}^{\infty}(\mathbb{R})$ we have that $T_{z} f(x)=\lim _{\epsilon \rightarrow 0^{+}} T_{\epsilon, z} f(x)$. Let $K_{\epsilon, z}$ be such that $T_{\epsilon, z} f(x)=K_{\epsilon, z} * f(x)$. To prove the $L^{2}$-boundedness of $T_{i b}$ as well as the $L^{1}$-boundedness of $T_{1+i b}$ on $H^{1}(\mathbb{R})$ we need some estimations of $\widehat{K_{\epsilon, i b}}$ and $\widehat{K_{\epsilon, 1+i b}}$, respectively. We devote this section to the proof of such estimates, which are contained in the following theorem. 
THEOREM 2.1. If $z=a+i b$ and $0 \leq a \leq 1$ then

$$
\left|\widehat{K_{\epsilon, z}(\xi)}\right| \leq \frac{C(1+|b|)}{\left(\sqrt{\left|\gamma^{\prime \prime}\left(\gamma^{\prime-1}(|\xi|)\right)\right|} \gamma^{\prime-1}(|\xi|)\right)^{a}}
$$

if $|\xi|$ is large, and

$$
\left|\widehat{K_{\epsilon, z}(\xi)}\right| \leq C(1+|b|)
$$

otherwise.

We start with a basic result on oscillatory integrals due to van der Corput.

LEMMA 2.1. Suppose that $\phi$ is real-valued and smooth on $(a, b)$, and that $\left|\phi^{k}(t)\right| \geq \lambda>0$ for all $t \in(a, b)$. Then

$$
\left|\int_{a}^{b} e^{i \phi(t)} d t\right| \leq C_{k} \lambda^{-1 / k}
$$

holds when:

(i) $k \geq 2$; or

(ii) $k=1$ and $\phi^{\prime}(t)$ is monotonic.

$C_{k}$ depends only on $k$.

The proof of Lemma 2.1 can be found in [4].

We now state some propositions needed to prove Theorem 2.1 .

Proposition 2.1. If $|\xi|$ is large, $0<\epsilon<\gamma^{\prime-1}(|\xi|)$, and $0 \leq a \leq 1$ then

$$
\int_{\epsilon}^{\gamma^{\prime-1}(|\xi|)}\left(\frac{t^{-1-a}}{\gamma^{\prime}(t) \psi(t)^{1-a}}+\frac{t^{-a}\left|\psi^{\prime}(t)\right|}{\gamma^{\prime}(t) \psi(t)^{2-a}}\right) d t \leq \frac{C}{\left(\sqrt{\left|\gamma^{\prime \prime}\left(\gamma^{\prime-1}(|\xi|)\right)\right|} \gamma^{\prime-1}(|\xi|)\right)^{a}}
$$

where $C$ is a constant independent of $|\xi|$ and $\epsilon$.

Proposition 2.2. If $|\xi|$ is large and $0 \leq a \leq 1$ then

$$
\frac{1}{|\xi|} \int_{\gamma^{\prime-1}(\xi \xi)}^{1}\left(\frac{\left|\psi^{\prime}(t)\right| t^{-a}}{\psi(t)^{2-a}}+\frac{t^{-1-a}}{\psi(t)^{1-a}}\right) d t \leq \frac{C}{\left(\sqrt{\left|\gamma^{\prime \prime}\left(\gamma^{\prime-1}(|\xi|)\right)\right|} \gamma^{\prime-1}(|\xi|)\right)^{a}}
$$

where $C$ is a constant independent of $|\xi|$.

Before we proceed to the proof of these propositions the following remarks are in order. 
REMARKS. (1) Given $u<v$ let $G(t)=\int_{u}^{t} e^{i(\gamma(s)-\xi s)} d s$ for $u<t<v$. Then $G^{\prime}(t)=e^{i(\gamma(t)-\xi t)}$. If $\rho(s)=\gamma(s)-\xi s$ then for any $u<s<t,\left|\rho^{\prime \prime}(s)\right|=\left|\gamma^{\prime \prime}(s)\right| \geq$ $\left|\gamma^{\prime \prime}(t)\right|$. Hence by Lemma 2.1 we have that $|G(t)| \leq C / \sqrt{\left|\gamma^{\prime \prime}(t)\right|}$.

(2) Since $\lim _{t \rightarrow 0^{+}} d\left(1 / \gamma^{\prime}(t)\right) / d t=0$, for $t>0$ close to zero $1 / \gamma^{\prime}(t) \leq C t$.

(3) Since $\gamma^{\prime}(x) \geq A \gamma^{\prime}((1+\epsilon) x)$ for $x$ small we have that $1 /\left(\gamma^{\prime-1}\left(A^{k+1}|\xi|\right)\right) \leq$ $(1+\epsilon) /\left(\gamma^{\prime-1}\left(A^{k}|\xi|\right)\right)$, for $|\xi|$ large and $k$ any positive integer.

(4) For $0 \leq a \leq 1$ and $t$ small we have that $\left|\gamma^{\prime \prime}(t)\right|^{a / 2} \leq C \gamma^{\prime}(t)^{a \lambda} \leq C \gamma^{\prime 1-\lambda(1-a)}(t)$. Hence $\left|\gamma^{\prime \prime}\left(\gamma^{\prime-1}(|\xi|)\right)\right|^{a / 2} \leq C|\xi|^{1-\lambda(1-a)}$ for $|\xi|$ large.

(5) If $t$ is small then $1 / t \leq \gamma^{\prime}(t)$. Hence if $|\xi|$ is large we must have that $1 / \gamma^{\prime-1}(|\xi|) \leq|\xi|$.

(6) For $|\xi|$ large, $1 /\left(\psi\left(\gamma^{\prime-1}(|\xi|)\right)\right) \leq C|\xi|$, since $1 / \psi(t) \leq C \sqrt{\left|\gamma^{\prime \prime}(t)\right|} \leq C \gamma^{\prime}(t)$.

(7) There exists a constant $C$ such that for $t>0$ close to zero, $1 / t \leq C\left|\gamma^{\prime \prime}(t)\right| / \gamma^{\prime}(t)$.

Remark 7 is a consequence of assumption (a.4):

$$
\epsilon a\left(-\gamma^{\prime \prime}(a)\right) \geq \int_{a}^{(1+\epsilon) a}-\gamma^{\prime \prime}(t) d t=\gamma^{\prime}(a)-\gamma^{\prime}((1+\epsilon) a) .
$$

Since

$$
-\gamma^{\prime}((1+\epsilon) a) \geq-\frac{\gamma^{\prime}(a)}{A}
$$

we have that

$$
\left|\gamma^{\prime \prime}(a)\right| \geq \frac{\gamma^{\prime}(a)-\gamma^{\prime}((1+\epsilon) a)}{\epsilon a} \geq \frac{\gamma^{\prime}(a)}{a}\left(\frac{A-1}{A}\right) \frac{1}{\epsilon}>\frac{\gamma^{\prime}(a)}{a} \frac{1}{A}
$$

which is Remark 7.

Let us prove the propositions.

PROOF OF PROPOSITION 2.1. Since $1 / \psi(t) \leq C \sqrt{\left|\gamma^{\prime \prime}(t)\right|} \leq C \gamma^{\prime}(t)^{\lambda}$ we see that

$$
\begin{aligned}
\int_{\epsilon}^{\gamma^{\prime-1}(|\xi|)} \frac{t^{-1-a}}{\gamma^{\prime}(t) \psi(t)^{1-a}} d t & \leq C \sum_{k=0}^{\infty} \int_{\gamma^{\prime-1}\left(A^{k+1}|\xi|\right)}^{\gamma^{\prime-1}\left(A^{k}|\xi|\right)} \frac{t^{-1-a} \gamma^{\prime}(t)^{\lambda(1-a)}}{\gamma^{\prime}(t)} d t \\
& \leq C \sum_{k=0}^{\infty} \frac{\left(A^{k}|\xi|\right)^{\lambda(1-a)}}{\left(\gamma^{\prime-1}\left(A^{k+1}|\xi|\right)\right)^{a} A^{k}|\xi|} \ln \left(\frac{\gamma^{\prime-1}\left(A^{k}|\xi|\right)}{\gamma^{\prime-1}\left(A^{k+1}|\xi|\right)}\right) \\
& \leq C \sum_{k=0}^{\infty} \frac{\left(A^{k}|\xi|\right)^{\lambda(1-a)}}{\left(\gamma^{\prime-1}\left(A^{k+1}|\xi|\right)\right)^{a} A^{k}|\xi|}
\end{aligned}
$$

since by Remark 3 we have that $\ln \left(\gamma^{\prime-1}\left(A^{k}|\xi|\right) / \gamma^{\prime-1}\left(A^{k+1}|\xi|\right)\right) \leq \ln (1+\epsilon)$. 
Hence iterating Remark $3 k$ times we see that

$$
\begin{aligned}
\int_{\epsilon}^{\gamma^{\prime-1}(|\xi|)} \frac{t^{-1-a}}{\gamma^{\prime}(t) \psi(t)^{1-a}} d t & \leq C \frac{|\xi|^{\lambda(1-a)}}{\left(\gamma^{\prime-1}(|\xi|)\right)^{a}|\xi|} \sum_{k=0}^{\infty}\left(\frac{1+\epsilon}{A}\right)^{k a} A^{k(\lambda-1)} \\
& \leq C\left(\gamma^{\prime-1}(|\xi|) \sqrt{\left|\gamma^{\prime \prime}\left(\gamma^{\prime-1}(|\xi|)\right)\right|}\right)^{-a} .
\end{aligned}
$$

The last inequality is due to Remark 4 and the facts that $1-\lambda>0$ and $1+\epsilon<A$.

Using assumption (a.2) we have that

$$
\begin{aligned}
\int_{\epsilon}^{\gamma^{\prime-1}(|\xi|)} \frac{t^{-a}\left|\psi^{\prime}(t)\right|}{\gamma^{\prime}(t) \psi(t)^{2-a}} d t & \leq C \sum_{k=0}^{\infty} \frac{\left(A^{k}|\xi|\right)^{\lambda(1-a)}}{\left(\gamma^{\prime-1}\left(A^{k+1}|\xi|\right)\right)^{a} A^{k}|\xi|} \int_{\gamma^{\prime-1}\left(A^{k+1}|\xi|\right)}^{\gamma^{\prime-1}\left(A^{k}|\xi|\right)}\left|\frac{\psi^{\prime}(t)}{\psi(t)}\right| d t \\
& \leq C \sum_{k=0}^{\infty} \frac{\left(A^{k}|\xi|\right)^{\lambda(1-a)}}{\left(\gamma^{\prime-1}\left(A^{k+1}|\xi|\right)\right)^{a} A^{k}|\xi|} \ln \left(\frac{\left|\gamma^{\prime \prime}\left(\gamma^{\prime-1}\left(A^{k+1}|\xi|\right)\right)\right|}{\left|\gamma^{\prime \prime}\left(\gamma^{\prime-1}\left(A^{k}|\xi|\right)\right)\right|}\right) .
\end{aligned}
$$

Using assumption (a.3) we see that there is a constant $C$ independent of $s$ so that

$$
\left|\gamma^{\prime \prime}\left(\gamma^{\prime-1}(A s)\right)\right| \leq C\left|\gamma^{\prime \prime}\left(\gamma^{\prime-1}(s)\right)\right|,
$$

and hence

$$
\ln \left(\frac{\left|\gamma^{\prime \prime}\left(\gamma^{\prime-1}\left(A^{k+1}|\xi|\right)\right)\right|}{\left|\gamma^{\prime \prime}\left(\gamma^{\prime-1}\left(A^{k}|\xi|\right)\right)\right|}\right) \leq C
$$

As before we have that

$$
\sum_{k=0}^{\infty} \frac{\left(A^{k}|\xi|\right)^{\lambda(1-a)}}{\left(\gamma^{\prime-1}\left(A^{k+1}|\xi|\right)\right)^{a} A^{k}|\xi|} \leq C\left(\gamma^{\prime-1}(|\xi|) \sqrt{\left|\gamma^{\prime \prime}\left(\gamma^{-1}(|\xi|)\right)\right|}\right)^{-a},
$$

and hence we have proved that

$$
\int_{\epsilon}^{\gamma^{\prime-1}(|\xi|)}\left(\frac{t^{-1-a}}{\gamma^{\prime}(t) \psi(t)^{1-a}}+\frac{t^{-a}\left|\psi^{\prime}(t)\right|}{\gamma^{\prime}(t) \psi(t)^{2-a}}\right) d t \leq \frac{C}{\left(\sqrt{\left|\gamma^{\prime \prime}\left(\gamma^{\prime-1}(|\xi|)\right)\right|} \gamma^{\prime-1}(|\xi|)\right)^{a}}
$$

ProOF OF PRoposition 2.2. Since $\psi$ is monotone and $1 / \psi(t) \leq C \sqrt{\left|\gamma^{\prime \prime}(t)\right|}$ we have that

$$
\begin{aligned}
\frac{1}{|\xi|} \int_{\gamma^{\prime-1}(|\xi|)}^{1} \frac{\left|\psi^{\prime}(t)\right| t^{-a}}{\psi(t)^{2-a}} d t & \leq \frac{C}{|\xi|} \frac{{\sqrt{\left|\gamma^{\prime \prime}\left(\gamma^{\prime-1}(|\xi|)\right)\right|}}^{1-a}}{\left(\gamma^{\prime-1}(|\xi|)\right)^{a}} \ln \left(\frac{\psi(1)}{\psi\left(\gamma^{\prime-1}(|\xi|)\right)}\right) \\
& \leq C\left(\sqrt{\left.\left|\gamma^{\prime \prime}\left(\gamma^{\prime-1}(|\xi|)\right)\right| \gamma^{\prime-1}(|\xi|)\right)^{-a}}\right.
\end{aligned}
$$


since

$$
\frac{\sqrt{\left|\gamma^{\prime \prime}\left(\gamma^{\prime-1}(|\xi|)\right)\right|} \ln \left(\psi(1) / \psi\left(\gamma^{\prime-1}(|\xi|)\right)\right)}{|\xi|} \leq C \frac{|\xi|^{\lambda} \ln (|\xi|)}{|\xi|} \leq C
$$

if $|\xi|$ is large.

Similarly

$$
\begin{aligned}
\frac{1}{|\xi|} \int_{\gamma^{\prime-1}(|\xi|)}^{1} \frac{t^{-1-a}}{\psi(t)^{1-a}} d t & \leq \frac{C}{|\xi|} \frac{\sqrt{\left|\gamma^{\prime \prime}\left(\gamma^{\prime-1}(|\xi|)\right)\right|}}{\left(\gamma^{\prime-1}(|\xi|)\right)^{a}} \ln \left(\frac{1}{\gamma^{\prime-1}(|\xi|)}\right) \\
& \leq C\left(\sqrt{\left|\gamma^{\prime \prime}\left(\gamma^{\prime-1}(|\xi|)\right)\right|} \gamma^{\prime-1}(|\xi|)\right)^{-a}
\end{aligned}
$$

since $\ln \left(1 / \gamma^{\prime-1}(|\xi|)\right) \leq C \ln (|\xi|)$.

Hence we have that

$$
\frac{1}{|\xi|} \int_{\gamma^{\prime-1}(\xi \mid)}^{1}\left(\frac{\left|\psi^{\prime}(t)\right| t^{-a}}{\psi(t)^{2-a}}+\frac{t^{-1-a}}{\psi(t)^{1-a}}+\right) d t \leq \frac{C}{\left(\sqrt{\left|\gamma^{\prime \prime}\left(\gamma^{\prime-1}(|\xi|)\right)\right|} \gamma^{\prime-1}(|\xi|)\right)^{a}}
$$

We can now proceed to the proof of Theorem 2.1.

Proof of Theorem 2.1. We need to estimate

$$
\widehat{K_{\epsilon, z}}(\xi)=\int_{\epsilon}^{1} e^{i(\gamma(t)-\xi t)} \frac{t^{-a-i b}}{\psi(t)^{1-a-i b}} d t
$$

where $z=a+i b$. In order to take advantage of the behavior of $\gamma$ near zero we would like to do an integration by parts by writing in the notation of Remark 1

$$
e^{i(\gamma(t)-\xi t)}=G^{\prime}(t)
$$

and then integrate by parts.

Case I: $\xi$ is small so that $\left|\gamma^{\prime}(t)-\xi\right| \geq C \gamma^{\prime}(t)$, for some constant $C$.

For $\epsilon<t<1$ we write $G(t)=\int_{\epsilon}^{t} e^{i(\gamma(s)-\xi s)} d s$. Since $\left|\rho^{\prime}(s)\right|=\left|\gamma^{\prime}(s)-\xi\right| \geq$ $C \gamma^{\prime}(s)$, using van der Corput's Lemma we see that $|G(t)| \leq C / \gamma^{\prime}(t)$.

We now have $\widehat{K_{\epsilon, z}}(\xi)=\int_{\epsilon}^{1} G^{\prime}(t) t^{-a-i b} / \psi(t)^{1-a-i b} d t$. We integrate by parts to get $\left|\widehat{K_{\epsilon, z}}(\xi)\right| \leq|\mathrm{I}|+\mid$ II $\mid$ where

$$
|\mathrm{I}| \leq \frac{C}{\gamma^{\prime}(1) \psi(1)} \leq C
$$


For II we have that

$$
\mathrm{II}=\int_{\epsilon}^{1} G(t) \frac{d}{d t}\left(\frac{t^{-a-i b}}{\psi(t)^{1-a-i b}}\right) d t
$$

and so

$$
|\mathrm{II}| \leq C(1+|b|) \int_{\epsilon}^{1}\left[\frac{t^{-a-1}}{\gamma^{\prime}(t) \psi(t)^{1-a}}+\frac{t^{-a}\left|\psi^{\prime}(t)\right|}{\psi(t)^{2-a} \gamma^{\prime}(t)}\right] d t
$$

Using Proposition 2.1 we see that $|\mathrm{II}| \leq C(1+|b|)$.

Case II: $|\xi|$ is large.

Let $t_{0}=\gamma^{\prime-1}(2|\xi|)$ and $t_{1}=\gamma^{\prime-1}(|\xi| / 2)$. We write

$$
\begin{aligned}
\widehat{K_{\epsilon, z}}(\xi) & =\int_{\epsilon}^{t_{0}}+\int_{t_{0}}^{t_{1}}+\int_{t_{1}}^{1} e^{i(\gamma(t)-\xi t)} \frac{t^{-a-i b}}{\psi(t)^{1-a-i b}} d t \\
& =\mathrm{I}+\mathrm{II}+\mathrm{III} .
\end{aligned}
$$

We now treat II, III, and I separately.

For $t_{0}<t<t_{1}$, define $G$ as in Remark $1, G(t)=\int_{t_{0}}^{t} e^{i(\gamma(s)-\xi s)} d s$. Then we have $|G(t)| \leq B / \sqrt{\left|\gamma^{\prime \prime}(t)\right|}$ and II $=\int_{t_{0}}^{t_{1}} G^{\prime}(t) t^{-a-i b} / \psi(t)^{1-a-i b} d t$. We integrate by parts to get

$$
|\mathrm{II}| \leq C\left(\left|\mathrm{II}_{1}\right|+\left|\mathrm{II}_{2}\right|\right)
$$

where

$$
\left|\mathrm{II}_{1}\right| \leq \frac{C}{\left(\sqrt{\left|\gamma^{\prime \prime}\left(t_{1}\right)\right|} t_{1}\right)^{a}}
$$

and

$$
\mathrm{II}_{2}=\int_{b_{0}}^{t_{1}} G(t) \frac{d}{d t}\left(\frac{t^{-a-i b}}{\psi(t)^{1-a-i b}}\right) d t
$$

So

$$
\left|\mathrm{II}_{2}\right| \leq C(1+|b|)\left(\int_{t_{0}}^{t_{1}} \frac{t^{-a}\left|\psi^{\prime}(t)\right|}{\psi(t)^{2-a} \sqrt{\left|\gamma^{\prime \prime}(t)\right|}} d t+\int_{t_{0}}^{t_{1}} \frac{t^{-1-a}}{\psi(t)^{1-a} \sqrt{\left|\gamma^{\prime \prime}(t)\right|}} d t\right) .
$$

Since $1 / \psi(t) \leq C \sqrt{\left|\gamma^{\prime \prime}(t)\right|}$ we see that

$$
\int_{t_{0}}^{t_{1}} \frac{t^{-1-a}}{\psi(t)^{1-a} \sqrt{\left|\gamma^{\prime \prime}(t)\right|}} d t \leq C \frac{1}{\left(t_{0} \sqrt{\left|\gamma^{\prime \prime}\left(t_{1}\right)\right|}\right)^{a}} \ln \left(\frac{t_{1}}{t_{0}}\right) .
$$


Since

$$
\frac{1}{\gamma^{\prime-1}(A|\xi|)} \leq \frac{1+\epsilon}{\gamma^{\prime-1}(|\xi|)}
$$

if $k$ is such that $2 / A^{k}<1$ we must have that

$$
\frac{1}{\gamma^{\prime-1}(2|\xi|)} \leq \frac{(1+\epsilon)^{k}}{\gamma^{\prime-1}\left(2|\xi| / A^{k}\right)} \leq \frac{(1+\epsilon)^{k}}{\gamma^{\prime-1}(|\xi|)}
$$

and so $\ln \left(t_{1} / t_{0}\right) \leq C$. Hence

$$
\int_{t_{0}}^{t_{1}} \frac{t^{-1-a}}{\psi(t)^{1-a} \sqrt{\left|\gamma^{\prime \prime}(t)\right|}} d t \leq C \frac{1}{\left(t_{0} \sqrt{\left|\gamma^{\prime \prime}\left(t_{1}\right)\right|}\right)^{a}} .
$$

Similarly, using assumptions (a.2) and (a.3) we get

$$
\int_{t_{0}}^{t_{1}} \frac{\left|\psi^{\prime}(t)\right| t^{-a}}{\psi(t)^{2-a} \sqrt{\left|\gamma^{\prime \prime}(t)\right|}} d t \leq \frac{1}{\left(t_{0} \sqrt{\left|\gamma^{\prime \prime}\left(t_{0}\right)\right|}\right)^{a}}\left|\ln \left(\frac{\gamma^{\prime \prime}\left(t_{0}\right)}{\gamma^{\prime \prime}\left(t_{1}\right)}\right)\right| \leq C\left(\frac{1}{\sqrt{\left|\gamma^{\prime \prime}\left(t_{0}\right)\right|}} \frac{1}{t_{0}}\right)^{a} \text {. }
$$

Finally, since

$$
\frac{1}{\gamma^{\prime-1}(2|\xi|)} \leq \frac{(1+\epsilon)^{k}}{\gamma^{\prime-1}(|\xi|)}
$$

and

$$
\left|\gamma^{\prime \prime}\left(\gamma^{\prime-1}(2 s)\right)\right| \leq C\left|\gamma^{\prime \prime}\left(\gamma^{\prime-1}(s)\right)\right|
$$

we get

$$
|\mathrm{II}| \leq \frac{C(1+|b|)}{\left(\sqrt{\left|\gamma^{\prime \prime}\left(\gamma^{\prime-1}(|\xi|)\right)\right|} \gamma^{\prime-1}(|\xi|)\right)^{a}} .
$$

Let's now estimate

$$
\mathrm{III}=\int_{t_{1}}^{1} e^{i(\gamma(t)-\xi t)} \frac{t^{-a-i b}}{\psi(t)^{1-a-i b}} d t .
$$

For $t_{1} \leq t \leq 1$ we define

$$
G(t)=\int_{t_{1}}^{t} e^{i(\gamma(s)-\xi s)} d s .
$$

For $t_{1} \leq s \leq t$ we have that $\left|\gamma^{\prime}(s)-\xi\right| \geq C|\xi|$ and $\gamma^{\prime}(s)-\xi$ is monotone, hence by van der Corput's Lemma we have that $|G(t)| \leq C /|\xi|$. 
We write

$$
\mathrm{III}=\int_{t_{1}}^{1} G^{\prime}(t) \frac{t^{-a-i b}}{\psi(t)^{1-a-i b}} d t
$$

and after an integration by parts we see that $\mathrm{III}=\mathrm{III}_{1}+\mathrm{III}_{2}$ with

$$
\left|\mathrm{III}_{1}\right| \leq \frac{C}{|\xi| \psi(1)^{1-a}} \leq \frac{C}{|\xi|} .
$$

Using Remark 4 we see that

$$
\left|\mathrm{III}_{1}\right| \leq C\left(\gamma^{\prime-1}(|\xi|) \sqrt{\left|\gamma^{\prime \prime}\left(\gamma^{\prime-1}(|\xi|)\right)\right|}\right)^{-a} .
$$

Since for $t_{1} \leq t \leq 1,|G(t)| \leq C /|\xi|$ we have that

$$
\left|\mathrm{III}_{2}\right| \leq \frac{C(1+|b|)}{|\xi|} \int_{t_{1}}^{1}\left[\frac{t^{-1-a}}{\psi(t)^{1-a}}+\frac{\left|\psi^{\prime}(t)\right| t^{-a}}{\psi(t)^{2-a}}\right] d t .
$$

By Proposition 2.2 we have that

$$
\left|\mathrm{III}_{2}\right| \leq C\left(\gamma^{\prime-1}(|\xi|) \sqrt{\left|\gamma^{\prime \prime}\left(\gamma^{\prime-1}(|\xi|)\right)\right|}\right)^{-a} .
$$

We still have to deal with

$$
\mathrm{I}=\int_{\epsilon}^{\phi_{0}} e^{i(\gamma(t)-\xi t)} \frac{t^{-a-i b}}{\psi(t)^{1-a-i b}} d t .
$$

For $\epsilon \leq t \leq t_{0}$ we define

$$
G(t)=\int_{\epsilon}^{t} e^{i(\gamma(s)-\xi s)} d s .
$$

In this case we have that $\left|\gamma^{\prime}(s)-\xi\right| \geq C \gamma^{\prime}(s)$ and hence we now have that $|G(t)| \leq$ $C / \gamma^{\prime}(t)$. We write

$$
\mathrm{I}=\int_{\epsilon}^{t_{0}} G^{\prime}(t) \frac{t^{-a-i b}}{\psi(t)^{1-a-i b}} d t
$$

and integrate by parts to get

$$
\begin{aligned}
|\mathrm{I}| & \leq \frac{C \sqrt{\left|\gamma^{\prime \prime}\left(t_{0}\right)\right|}}{\gamma^{\prime}\left(t_{0}\right)\left(\sqrt{\left|\gamma^{\prime \prime}\left(t_{0}\right)\right|} t_{0}\right)^{a}}+\left|\int_{\epsilon}^{t_{0}} G(t) \frac{d}{d t}\left(\frac{t^{-a-i b}}{\psi(t)^{1-a-i b}}\right) d t\right| \\
& =\mathrm{I}_{1}+\mathrm{I}_{2} .
\end{aligned}
$$


As before, we have that

$$
\mathrm{I}_{1} \leq \frac{C}{\left(\sqrt{\left|\gamma^{\prime \prime}\left(\gamma^{\prime-1}(|\xi|)\right)\right|} \gamma^{\prime-1}(|\xi|)\right)^{a}} .
$$

Since for $\epsilon \leq t \leq t_{0}$ we have that $|G(t)| \leq \frac{c}{\gamma^{\prime}(t)}$, we see that

$$
\mathrm{I}_{2} \leq C(1+|b|) \int_{\epsilon}^{t_{0}}\left[\frac{t^{-1-a}}{\gamma^{\prime}(t) \psi(t)^{1-a}}+\frac{\left|\psi^{\prime}(t)\right| t^{-a}}{\gamma^{\prime}(t) \psi(t)^{2-a}}\right] d t .
$$

In view of Proposition 2.1 we can conclude that

$$
\mathrm{I}_{2} \leq C\left(\gamma^{\prime-1}(|\xi|) \sqrt{\left|\gamma^{\prime \prime}\left(\gamma^{\prime-1}(|\xi|)\right)\right|}\right)^{-a}
$$

So for $|\xi|$ small

$$
\left|\widehat{K_{\epsilon, z}}(\xi)\right| \leq C(1+|b|)
$$

and for $|\xi|$ large

$$
\left|\widehat{K_{\epsilon, z}}(\xi)\right| \leq \frac{C(1+|b|)}{\left(\sqrt{\left.\left|\gamma^{\prime \prime}\left(\gamma^{\prime-1}|\xi|\right)\right| \gamma^{\prime-1}(|\xi|)\right)^{a}}\right.}
$$

and Theorem 2.1 is proved.

\section{3. $L^{p}(\mathbb{R})$-boundedness of $T_{\theta}$}

In this section we want to prove the statement of the $L^{p}$-boundedness of $T_{\theta}$, that is Theorem 1.3. We start with the proof of Theorem 1.4 and Theorem 1.5. Then using an interpolation argument we will be able to prove Theorem 1.3.

3.1. $L^{2}(\mathbb{R})$-boundedness of $T_{i b} \quad$ To prove the $L^{2}$-boundedness of $T_{i b}$ it is enough to prove the following theorem.

THEOREM 3.1. $\left\|T_{\epsilon, i b} f\right\|_{L^{2}} \leq C(1+|b|)\|f\|_{L^{2}}$ for every $f$ in $C_{0}^{\infty}(\mathbb{R})$. The constant $C$ is independent of $\epsilon$.

To prove this statement we just have to see that $\left|\widehat{K_{\epsilon, i b}}(\xi)\right| \leq C(1+|b|)$, where $C$ is independent of $\xi$ and $\epsilon$. Since this is Theorem 2.1 when $a=0$, there is nothing to prove. 
However it is important to mention the following. To prove that $T_{i b}$ is bounded on $L^{2}(\mathbb{R})$ it is enough to assume that $1 / \psi(t) \leq C \sqrt{\left|\gamma^{\prime \prime}(t)\right|}$.

The stronger assumption (a.2)

$$
\left|\frac{\psi^{\prime}(t)}{\psi(t)}\right| \leq C\left|\frac{\gamma^{\prime \prime \prime}(t)}{\gamma^{\prime \prime}(t)}\right|
$$

was used twice during the proof of Theorem 2.1, to estimate a pair of integrals, namely

$$
\int_{\epsilon}^{\gamma^{\prime-1}(|\xi|)} \frac{t^{-a}\left|\psi^{\prime}(t)\right|}{\gamma^{\prime}(t) \psi(t)^{2-a}} d t \text { and } \int_{t_{0}}^{t_{1}} \frac{t^{-a}\left|\psi^{\prime}(t)\right|}{\sqrt{\left|\gamma^{\prime \prime}(t)\right| \psi(t)^{2-a}}} d t .
$$

It can be easily seen that when $a=0$ the desired estimate for these two integrals follows from the monotonicity of $\psi$ and the weaker assumption $1 / \psi(t) \leq C \sqrt{\left|\gamma^{\prime \prime}(t)\right|}$.

3.2. $L^{1}(\mathbb{R})$-boundedness of $T_{1+i b}$ on $H^{1}(\mathbb{R})$ Again, to prove Theorem 1.5 it is enough to prove the following theorem.

THEOREM 3.2. $\left\|T_{\epsilon, 1+i b} f\right\|_{L^{\prime}} \leq C(1+|b|)\|f\|_{H^{\prime}}$ for every $f$ in $H_{1}(\mathbb{R})$. The constant $C$ is independent of $\epsilon$.

Since $K_{\epsilon, 1+i b}$ is the kernel of $T_{\epsilon, 1+i b}$ we have that

$$
K_{\epsilon, 1+i b}(x)= \begin{cases}\frac{e^{i \gamma(x)}}{x^{1+i b} \psi(x)^{-i b}} & \text { if } \epsilon \leq x \leq 1 \\ 0 & \text { otherwise. }\end{cases}
$$

Theorem 3.2 will be a consequence of the following Calderón-Zygmund type lemmas. The first one is Theorem 2.1 for $a=1$. We restate it here for convenience.

LEMMA 3.1. For $\xi$ large $\left|\widehat{X_{\epsilon, 1+i b}}(\xi)\right| \leq(C(1+|b|)) /\left(\sqrt{\left|\gamma^{\prime \prime}\left(\gamma^{\prime-1}(|\xi|)\right)\right|} \gamma^{\prime-1}(|\xi|)\right)$, and for $\xi$ small $\left|\widehat{K_{\epsilon, 1+i b}}(\xi)\right| \leq C(1+|b|)$. The constant $C$ is independent of $\epsilon$.

LEMMA 3.2. For $L$ small and $|y| \leq L$

$$
\int_{|x| \geq 2 \gamma^{\prime-1}\left(|y|^{-1}\right)}\left|K_{\epsilon, 1+i b}(x-y)-K_{\epsilon, 1+i b}(x)\right| d x \leq C(1+|b|) .
$$

Let's assume for a moment Lemma 3.2 in order to prove Theorem 3.2.

PROOF OF THEOREM 3.2. It is enough to do it for atoms. So let $a(x)$ be an atom. Without loss of generality we can assume that $a$ is supported in $(-L, L)$. Since $a$ is an atom we have that $|a(x)| \leq 1 / L$ and $\int a(x) d x=0$. 
Case I: $L \geq 1$. Using Schwarz's inequality we have

$$
\begin{aligned}
\int\left|T_{\epsilon, 1+i b} a(x)\right| d x & \leq \int_{-L-1}^{L+1}\left|T_{\epsilon, 1+i b} a(x)\right| d x \\
& \leq C(L+1)^{1 / 2}\left(\int\left|T_{\epsilon, 1+i b} a(x)\right|^{2} d x\right)^{1 / 2} \\
& \leq C(1+|b|)(L+1)^{1 / 2}\left(\int|a(x)|^{2} d x\right)^{1 / 2} .
\end{aligned}
$$

The last inequality follows from the fact that $T_{\epsilon, 1+i b}$ is bounded in $L^{2}(\mathbb{R})$, by Lemma 3.1 .

Since $\int|a(x)|^{2} d x \leq 1 / L$ and $L \geq 1$ we get

$$
\int\left|T_{\epsilon, 1+i b} a(x)\right| d x \leq C(1+|b|)\left(\frac{L+1}{L}\right)^{1 / 2} \leq C(1+|b|) .
$$

Case II: $L<1$. Let $L$ be as in Lemma 3.2 and write

$$
\begin{aligned}
\int\left|T_{\epsilon, 1+i b} a(x)\right| d x & =\int_{|x| \leq 2 \gamma^{\prime-1}(1 / L)}\left|T_{\epsilon, 1+i b} a(x)\right| d x+\int_{|x| \geq 2 \gamma^{\prime-1}(1 / L)}\left|T_{\epsilon, 1+i b} a(x)\right| d x \\
& =\mathrm{I}+\mathrm{II} .
\end{aligned}
$$

We will first estimate I. Again using Schwarz's inequality we have

$$
\begin{aligned}
\mathrm{I}^{2} \leq & 2 \gamma^{\prime-1}\left(\frac{1}{L}\right) \int\left|T_{\epsilon, 1+i b} a(x)\right|^{2} d x \\
= & 2 \gamma^{\prime-1}\left(\frac{1}{L}\right) \int\left|\widehat{K_{\epsilon, 1+i b}}(\xi) \widehat{a}(\xi)\right|^{2} d \xi \\
= & 2 \gamma^{\prime-1}\left(\frac{1}{L}\right)\left[\int_{|\xi| \leq 1}\left|\widehat{K_{\epsilon, 1+i b}}(\xi) \widehat{a}(\xi)\right|^{2} d \xi\right. \\
& \left.+\int_{1 \leq|\xi| \leq \frac{1}{L}}\left|\widehat{K_{\epsilon, 1+i b}}(\xi) \widehat{a}(\xi)\right|^{2} d \xi+\int_{|\xi| \geq \frac{1}{L}}\left|\widehat{K_{\epsilon, 1+i b}}(\xi) \widehat{a}(\xi)\right|^{2} d \xi\right] \\
= & \mathrm{I}_{1}+\mathrm{I}_{2}+\mathrm{I}_{3} .
\end{aligned}
$$

Since $\left|\widehat{K_{\epsilon, 1+i b}}(\xi)\right| \leq C(1+|b|)$ for $\xi$ small and $|\widehat{a}(\xi)| \leq\|a\|_{L^{1}} \leq 1$ we have

$$
\begin{aligned}
\left|I_{1}\right| & \leq 2 \gamma^{\prime-1}\left(\frac{1}{L}\right) C(1+|b|)^{2} \int_{|\xi| \leq 1}|\widehat{a}(\xi)|^{2} d \xi \\
& \leq 2 \gamma^{\prime-1}\left(\frac{1}{L}\right) C(1+|b|)^{2} \leq C(1+|b|)^{2} .
\end{aligned}
$$


By Lemma 3.1 and the fact that $|\widehat{a}(\xi)| \leq 1$ we see that

$$
\left|\mathrm{I}_{2}\right| \leq 2 \gamma^{\prime-1}\left(\frac{1}{L}\right) C(1+|b|)^{2} \int_{1 \leq|\xi| \leq \frac{1}{L}} \frac{d \xi}{\left|\gamma^{\prime \prime}\left(\gamma^{\prime-1}(|\xi|)\right)\right|\left(\gamma^{\prime-1}(|\xi|)\right)^{2}} .
$$

Make the change of variable $u=\gamma^{\prime-1}(\xi)$ to get

$$
\begin{aligned}
\left|\mathbf{I}_{2}\right| & \leq 2 \gamma^{\prime-1}\left(\frac{1}{L}\right) C(1+|b|)^{2} \int_{\gamma^{\prime-1}(1 / L) \leq u \leq \gamma^{\prime-1}(1)} u^{-2} d u \\
& \leq C(1+|b|)^{2} \gamma^{\prime-1}\left(\frac{1}{L}\right)\left[\frac{1}{\gamma^{\prime-1}(1)}+\frac{1}{\gamma^{\prime-1}(1 / L)}\right] \leq C(1+|b|)^{2} .
\end{aligned}
$$

Since for $\xi$ large

$$
\left|\widehat{K_{\epsilon, 1+i b}}(\xi)\right| \leq \frac{C(1+|b|)}{\sqrt{\left|\gamma^{\prime \prime}\left(\gamma^{\prime-1}(|\xi|)\right)\right| \gamma^{\prime-1}(|\xi|)}}
$$

and for $t$ small $\gamma^{\prime}(t) / t \leq C\left|\gamma^{\prime \prime}(t)\right|$, we see that for $\xi$ large

$$
\left|\widehat{K_{\epsilon, 1+i b}}(\xi)\right|^{2} \leq \frac{C(1+|b|)^{2}}{|\xi| \gamma^{\prime-1}(|\xi|)} \text {. }
$$

This together with the fact that $\int|\widehat{a}(\xi)|^{2} d \xi=\int|a(x)|^{2} d x \leq 1 / L$ gives us

$$
\begin{aligned}
\left|\mathrm{I}_{3}\right| & \leq 2 \gamma^{\prime-1}\left(\frac{1}{L}\right) \sum_{k=0}^{\infty} \int_{\frac{A^{k}}{L} \leq|\xi| \leq \frac{A^{k+1}}{L}}\left|\widehat{K_{\epsilon, 1+i b}}(\xi) \widehat{a}(\xi)\right|^{2} d \xi \\
& \leq 2 C(1+|b|)^{2} \gamma^{\prime-1}\left(\frac{1}{L}\right) \sum_{k=0}^{\infty} \frac{L}{A^{k} \gamma^{\prime-1}\left(A^{k+1} / L\right)} \int|\widehat{a}(\xi)|^{2} d \xi \\
& \leq 2 C(1+|b|)^{2} \gamma^{\prime-1}\left(\frac{1}{L}\right) \sum_{k=0}^{\infty} \frac{1}{A^{k} \gamma^{\prime-1}\left(A^{k+1} / L\right)} .
\end{aligned}
$$

Now by hypothesis we have that for $x$ small $\gamma^{\prime}(x) \geq A \gamma^{\prime}((1+\epsilon) x)$. For $t$ large let $x=\gamma^{\prime-1}(t)$. We then get

$$
\begin{array}{cl} 
& \frac{t}{A} \geq \gamma^{\prime}\left((1+\epsilon) \gamma^{\prime-1}(t)\right) \\
\text { if and only if } & \gamma^{\prime-1}\left(\frac{t}{A}\right) \leq(1+\epsilon) \gamma^{\prime-1}(t) \\
\text { if and only if } & \gamma^{\prime-1}(\xi) \leq(1+\epsilon) \gamma^{\prime-1}(A \xi) .
\end{array}
$$

So finally we have

$$
\begin{aligned}
\left|I_{3}\right| & \leq 2 C(1+|b|)^{2} \gamma^{\prime-1}\left(\frac{1}{L}\right) \sum_{k=0}^{\infty} \frac{(1+\epsilon)^{k+1}}{A^{k} \gamma^{\prime-1}(1 / L)} \\
& =2 C(1+|b|)^{2}(1+\epsilon) \sum_{k=0}^{\infty}\left(\frac{1+\epsilon}{A}\right)^{k} \leq C(1+|b|)
\end{aligned}
$$


since $1+\epsilon<A$.

So we do have that $|\mathrm{I}| \leq C(1+|b|)$. It remains to see that $|\mathrm{II}| \leq C(1+|b|)$, where II $=\int_{|x| \geq 2 \gamma^{\prime-1}(1 / L)}\left|T_{\epsilon, 1+i b} a(x)\right| d x$.

Since $\int a(x) d x=0$ we have

$$
T_{\epsilon, 1+i b} a(x)=\int K_{\epsilon, 1+i b}(x-y) a(y) d y=\int\left[K_{\epsilon, 1+i b}(x-y)-K_{\epsilon, 1+i b}(x)\right] a(y) d y .
$$

Hence

$$
\begin{aligned}
|\mathrm{II}| & \leq \int_{|x| \geq 2 \gamma^{\prime-1}(1 / L)}\left|\int\left[K_{\epsilon, 1+i b}(x-y)-K_{\epsilon, 1+i b}(x)\right] a(y) d y\right| d x \\
& \leq \int|a(y)| d y \int_{|x| \geq 2 \gamma^{\prime-1}(1 / L)}\left|K_{\epsilon, 1+i b}(x-y)-K_{\epsilon, 1+i b}(x)\right| d x \\
& \leq \int|a(y)| d y \int_{|x| \geq 2 \gamma^{\prime-1}\left(\left.|y|\right|^{-1)}\right.}\left|K_{\epsilon, 1+i b}(x-y)-K_{\epsilon, 1+i b}(x)\right| d x \\
& \leq C(1+|b|) \int|a(y)| d y \leq C(1+|b|) .
\end{aligned}
$$

The next to last inequality is due to Lemma 3.2.

Altogether we have that $\int\left|T_{\epsilon, 1+i b} a(x)\right| d x \leq C(1+|b|)$ and Theorem 3.2 is proved.

Let's now prove Lemma 3.2.

PROOF OF LEMMA 3.2. Recall that

$$
K_{\epsilon, 1+i b}(x)= \begin{cases}\frac{e^{i \gamma(x)}}{x^{1+i b} \psi(x)^{-i b}} & \text { if } \epsilon \leq x \leq 1 \\ 0 & \text { otherwise. }\end{cases}
$$

For $0 \leq t \leq 1$ let $f(t)=K_{\epsilon, 1+i b}(x-t y)$. Then

$$
K_{\epsilon, 1+i b}(x-y)-K_{\epsilon, 1+i b}(x)=\int_{0}^{1} f^{\prime}(t) d t=\int_{0}^{1}-y K_{\epsilon, 1+i b}^{\prime}(x-t y) d t .
$$

So

$$
\begin{aligned}
\left|K_{\epsilon, 1+i b}(x-y)-K_{\epsilon, 1+i b}(x)\right| \leq & |y|(1+|b|) \int_{0}^{1}\left|\frac{\gamma^{\prime}(x-t y)}{x-t y}\right| d t \\
& +|y|(1+|b|) \int_{0}^{1} \frac{1}{|x-t y|^{2}} d t \\
& +|y|(1+|b|) \int_{0}^{1}\left|\frac{\psi^{\prime}(x-t y)}{\psi(x-t y)}\right| \frac{1}{|x-t y|} d t .
\end{aligned}
$$


And hence

$$
\begin{aligned}
& \int\left|K_{\epsilon, 1+i b}(x-y)-K_{\epsilon, 1+i b}(x)\right| d x \\
& \leq|y|(1+|b|) \iint_{0}^{1}\left|\frac{\gamma^{\prime}(x-t y)}{x-t y}\right| d t d x+|y|(1+|b|) \iint_{0}^{1} \frac{1}{|x-t y|^{2}} d t d x \\
& \quad+|y|(1+|b|) \iint_{0}^{1}\left|\frac{\psi^{\prime}(x-t y)}{\psi(x-t y)}\right| \frac{1}{|x-t y|} d t d x .
\end{aligned}
$$

Making the change of variable $z=x-t y$ and interchanging the order of integration we get

$$
\begin{aligned}
& \int_{|x| \geq 2 \gamma^{\prime-1}\left(|y|^{-1}\right)}\left|K_{\epsilon, 1+i b}(x-y)-K_{\epsilon, 1+i b}(x)\right| d x \\
& \leq|y|(1+|b|) \int_{1 \geq|z| \geq 2 \gamma^{\prime-1}\left(|y|^{-1}\right)-|y|} \gamma^{\prime}(z) \frac{d z}{|z|}+|y|(1+|b|) \int_{1 \geq|z| \geq 2 \gamma^{\prime-1}\left(|y|^{-1}\right)-|y|} \frac{d z}{|z|^{2}} \\
& \quad+|y|(1+|b|) \int_{1 \geq|z| \geq 2 \gamma^{\prime-1}\left(|y|^{-1}\right)-|y|}\left|\frac{\psi^{\prime}(z)}{z \psi(z)}\right| d z=\mathrm{I}+\mathrm{II}+\mathrm{III} .
\end{aligned}
$$

For $L$ small and $|y| \leq L$ we have that $1 /|y| \leq \gamma^{\prime}(|y|)$. Hence $|y| / \gamma^{\prime-1}\left(|y|^{-1}\right) \leq 1$ and $2 \gamma^{\prime-1}\left(|y|^{-1}\right)-|y| \geq \gamma^{\prime-1}\left(|y|^{-1}\right)$. So

$$
\mathrm{II} \leq C|y|(1+|b|)\left(\frac{1}{2}+\frac{1}{2 \gamma^{\prime-1}\left(|y|^{-1}\right)-|y|}\right) \leq C(1+|b|) .
$$

Since $\gamma^{\prime}(x) / x \leq C\left|\gamma^{\prime \prime}(x)\right|$ we see that

$$
\begin{aligned}
I & \leq|y|(1+|b|) \int_{1 \geq|z| \geq 2 \gamma^{\prime-1}\left(|y|^{-1}\right)-|y|}\left(-\gamma^{\prime \prime}(z)\right) d z \\
& \leq|y|(1+|b|)\left[\gamma^{\prime}\left(2 \gamma^{\prime-1}\left(|y|^{-1}\right)-|y|\right)+\gamma^{\prime}(1)\right] .
\end{aligned}
$$

Since $2 \gamma^{\prime-1}\left(|y|^{-1}\right)-|y| \geq \gamma^{\prime-1}\left(|y|^{-1}\right)$ we have that $\gamma^{\prime}\left(2 \gamma^{\prime-1}\left(|y|^{-1}\right)-|y|\right) \leq|y|^{-1}$.

So I $\leq C(1+|b|)$. To estimate III it is sufficient to note that

$$
\left|\frac{\psi^{\prime}(z)}{z \psi(z)}\right| \leq C \frac{1}{|z \psi(z)|} \leq C \frac{\sqrt{\left|\gamma^{\prime \prime}(z)\right|}}{|z|} \leq C \frac{\gamma^{\prime}(z)}{z} .
$$

So III $\leq C$ I $\leq C(1+|b|)$. Altogether we have

$$
\int_{|x| \geq 2 \gamma^{\prime-1}\left(|y|^{-1}\right)}\left|K_{\epsilon, 1+i b}(x-y)-K_{\epsilon, 1+i b}(x)\right| d x \leq C(1+|b|),
$$

for $|y|<L$ and Lemma 3.2 is proved. 
3.3. $L^{p}(\mathbb{R})$-boundedness of $T_{\theta}$ To prove Theorem 1.3 we will need a theorem on interpolation of analytic families of operators. Here we will formulate the version needed to prove the desired $L^{p}$-estimate. The proof of this particular case can be found in [1]. For the general version see [5].

Let $S$ be the open strip of complex numbers $z$ such that $0<\operatorname{Re}(z)<1$. Consider the mapping taking $z$ to $T_{z}$ from the closure of $S$ to bounded operators on $L^{2}(\mathbb{R})$. Suppose this mapping is analytic in $S$ and continuous and bounded in the closure of $S$. Then we have the following theorem.

THEOREM 3.3. Suppose $\left\|T_{i y} f\right\|_{L^{\prime}} \leq M_{0}(y)\|f\|_{H^{\prime}}$ for $f \in L^{2}(\mathbb{R}) \cap H^{1}(\mathbb{R})$ and $\left\|T_{1+i y} f\right\|_{L^{2}} \leq M_{1}(y)\|f\|_{L^{2}}$ for $f \in L^{2}(\mathbb{R})$, where $M_{i}(y) \leq A_{i}(1+|y|)^{N}$ for some $N$, and $i=0$, 1. Then $\left\|T_{t} f\right\|_{L^{p}} \leq M_{t}\|f\|_{L^{p}}$ for $f \in L^{2}(\mathbb{R}) \cap L^{p}(\mathbb{R})$ whenever $0<t<1$ and $1 / p=1-t / 2$. $M_{t}$ depends only on $t, A_{0}$ and $A_{1}$.

Taking $\theta=1-t$ in Theorem 3.3 we see that

$$
T_{\theta} f(x)=\lim _{\epsilon \rightarrow 0^{+}} \int_{\epsilon}^{1} e^{i \gamma(t)} f(x-t) \frac{t^{-\theta}}{\psi(t)^{1-\theta}} d t
$$

is bounded on $L^{p}(\mathbb{R})$ for $1 / p=(1+\theta) / 2,0 \leq \theta<1$. This concludes the proof of the $L^{p}$-boundedness of $T_{\theta}$.

\section{Sharp $L^{2}(\mathbb{R})$ result}

The purpose of this section is to prove that if

$$
\lim _{t \rightarrow 0^{+}} \frac{1}{\left|\gamma^{\prime \prime}(t)\right| \psi^{2}(t)}=\infty
$$

and if there is a constant $A \geq 1$ such that for all large $s>0$

$$
\gamma^{\prime}\left(|\gamma|^{-1}(2 s)\right) \leq A \gamma^{\prime}\left(|\gamma|^{-1}(s)\right)
$$

then $T_{0}$ cannot be bounded on $L^{2}(\mathbb{R})$.

In order to prove this we will need to find a lower bound for $\left|T_{0} f(x)\right|$ for certain points $x$ and an appropriate function $f$ in $L^{2}(\mathbb{R})$. These points $x$ will be such that for $t$ close to $x$, the oscillation $e^{i \gamma(t)}$ will not vary too much. Those points will lie in the intervals built in the following lemma.

LEMMA 4.1. Suppose there is a constant $A \geq 1$ such that for all large $s>0$

$$
\gamma^{\prime}\left(|\gamma|^{-1}(2 s)\right) \leq A \gamma^{\prime}\left(|\gamma|^{-1}(s)\right) .
$$

Then there is a constant $B_{0}$ such that whenever $\epsilon \leq B_{0} \leq 1$ and $k \leq|\gamma|(\epsilon)$, the following is true: 
(i)

$$
\frac{B}{\gamma^{\prime}(\epsilon)} \leq|\gamma|^{-1}\left(2 k \pi-\frac{\pi}{3}\right)-|\gamma|^{-1}\left(2 k \pi+\frac{\pi}{3}\right)
$$

where $B=2 \pi /\left(3 A^{3}\right)$;

(ii) Let $I_{k}=\left[|\gamma|^{-1}(2 k \pi+\pi / 3)+B /\left(3 \gamma^{\prime}(\epsilon)\right),|\gamma|^{-1}(2 k \pi-\pi / 3)-B /\left(3 \gamma^{\prime}(\epsilon)\right)\right]$ and $J_{k}=\left[|\gamma|^{-1}(2(k+1) \pi-\pi / 3)-B /\left(3 \gamma^{\prime}(\epsilon)\right),|\gamma|^{-1}(2 k \pi+\pi / 3)+B /\left(3 \gamma^{\prime}(\epsilon)\right)\right]$. If $k^{\prime}$ is such that $k^{\prime} \leq|\gamma|(\epsilon)<k^{\prime}+1$ then

$$
\left(\epsilon,|\gamma|^{-1}(7)\right) \subseteq I_{k^{\prime}} \cup\left[\bigcup_{k=1}^{k^{\prime}-1} J_{k} \cup I_{k}\right] .
$$

(iii) There is a constant $D$ such that $\left|J_{k}\right| \leq D\left|I_{k+1}\right|$.

The construction of these intervals when the phase function is the inverse of a power can be found in [3].

We will also need the following fact. If $\gamma$ is such that $\gamma^{\prime}(t) \geq 0$ for $t>0, \gamma^{\prime}$ is decreasing, $\lim _{\epsilon \rightarrow 0^{+}} \gamma^{\prime}(\epsilon)=\infty$ and $\lim _{\epsilon \rightarrow 0^{+}} \gamma^{\prime \prime}(\epsilon) / \gamma^{\prime}(\epsilon)^{2}=0$, then

$$
\lim _{\epsilon \rightarrow 0^{+}} \gamma^{\prime}\left(\epsilon+1 / \gamma^{\prime}(\epsilon)\right) / \gamma^{\prime}(\epsilon)=1
$$

To see this we write

$$
\begin{aligned}
\gamma^{\prime}\left(\epsilon+\frac{1}{\gamma^{\prime}(\epsilon)}\right) & =\gamma^{\prime}\left(\epsilon+\frac{1}{\gamma^{\prime}(\epsilon)}\right)+\gamma^{\prime}(\epsilon)-\gamma^{\prime}(\epsilon) \\
& =\gamma^{\prime}(\epsilon)+\int_{\epsilon}^{\epsilon+1 / \gamma^{\prime}(\epsilon)} \gamma^{\prime \prime}(t) d t .
\end{aligned}
$$

Since

$$
\left|\int_{\epsilon}^{\epsilon+1 / \gamma^{\prime}(\epsilon)} \gamma^{\prime \prime}(t) d t\right| \leq \frac{\left|\gamma^{\prime \prime}(\epsilon)\right|}{\gamma^{\prime}(\epsilon)}
$$

and $\lim _{\epsilon \rightarrow 0^{+}} \gamma^{\prime \prime}(\epsilon) / \gamma^{\prime}(\epsilon)^{2}=0$ we get

$$
\lim _{\epsilon \rightarrow 0^{+}} \frac{\gamma^{\prime}\left(\epsilon+1 / \gamma^{\prime}(\epsilon)\right)}{\gamma^{\prime}(\epsilon)}=1
$$

Let's assume Lemma 4.1 and prove Theorem 1.6.

PROOF OF THEOREM 1.6. We are going to argue by contradiction. Suppose that $T_{0}$ is bounded on $L^{2}(\mathbb{R})$ and that $\lim _{t \rightarrow 0^{+}} 1 /\left(|\psi(t)| \sqrt{\left|\gamma^{\prime \prime}(t)\right|}\right)=\infty$. Then there exists a positive, decreasing function $g$ such that $1 / \psi^{2}(t) \geq\left|\gamma^{\prime \prime}(t)\right| g(t)$ and $\lim _{t \rightarrow 0^{+}} g(t)=\infty$. 
Let $f(x)=1$ for $x \in(-1,1)$ and 0 otherwise so that $\int|f(x)| d x=2$ and $\int|f(x)|^{2} d x=2$. Let $\epsilon$ and $B$ be the same as in Lemma 4.1 and define

$$
f_{\epsilon}(x)=f\left(\frac{x}{B /\left(3 \gamma^{\prime}(\epsilon)\right)}\right) .
$$

In the notation of Lemma 4.1 let $x \in I_{k}$. If $x \in I_{k}$ and $|x-t| \leq B /\left(3 \gamma^{\prime}(\epsilon)\right)$ then

$$
2 k \pi-\frac{\pi}{3} \leq|\gamma|(t) \leq 2 k \pi+\frac{\pi}{3} .
$$

Hence for $0 \leq \epsilon^{\prime} \leq \epsilon$ and $x \in I_{k}$ we have

$$
\begin{aligned}
\left|\int_{\epsilon^{\prime}}^{1} e^{i \gamma(t)} f_{\epsilon}(x-t) \frac{d t}{\psi(t)}\right| & \geq \frac{1}{2} \int_{\epsilon^{\prime}}^{1} f_{\epsilon}(x-t) \frac{d t}{\psi(t)} \\
& \geq \frac{1}{2} \int_{\epsilon^{\prime}}^{1} f_{\epsilon}(x-t) \frac{d t}{\psi\left(x+B /\left(3 \gamma^{\prime}(\epsilon)\right)\right)} .
\end{aligned}
$$

The last inequality is due to the fact that since

$$
t=|t| \leq|x-t|+x \leq \frac{B}{3 \gamma^{\prime}(\epsilon)}+x
$$

and $\psi$ is monotone then $\psi(t) \leq \psi\left(B /\left(3 \gamma^{\prime}(\epsilon)\right)+x\right)$.

So we have

$$
\begin{aligned}
\left|\int_{\epsilon^{\prime}}^{1} e^{i \gamma(t)} f_{\epsilon}(x-t) \frac{d t}{\psi(t)}\right| & \geq \frac{1}{2} \frac{1}{\psi\left(x+B /\left(3 \gamma^{\prime}(\epsilon)\right)\right)} \int f_{\epsilon}(x-t) d t \\
& =\frac{B}{6 \psi\left(x+B /\left(3 \gamma^{\prime}(\epsilon)\right)\right) \gamma^{\prime}(\epsilon)} .
\end{aligned}
$$

Thus for $x \in I_{k}$ we have

$$
\left|T_{0} f_{\epsilon}(x)\right| \geq \frac{C}{\gamma^{\prime}(\epsilon) \psi\left(B /\left(3 \gamma^{\prime}(\epsilon)\right)+x\right)} .
$$

Hence we get

$$
\int_{I_{k}}\left|T_{0} f_{\epsilon}(x)\right|^{2} d x \geq \frac{C}{\gamma^{\prime}(\epsilon)^{2}} \int_{I_{k}} \frac{d x}{\psi\left(B /\left(3 \gamma^{\prime}(\epsilon)\right)+x\right)^{2}} .
$$

And so we see that

$$
\int\left|T_{0} f_{\epsilon}(x)\right|^{2} d x \geq \frac{C}{\gamma^{\prime}(\epsilon)^{2}} \sum_{k=1}^{k^{\prime}} \int_{I_{k}} \frac{d x}{\psi\left(B /\left(3 \gamma^{\prime}(\epsilon)\right)+x\right)^{2}} .
$$


For any $1 \leq l \leq k^{\prime}-1$, we have $\left|J_{l}\right| \leq D\left|I_{l+1}\right|$. Since $\psi$ is monotone and $I_{l+1}$ lies to the left of $J_{l}$ we have that $\max _{J_{l}} 1 / \psi(t) \leq \min _{I_{t+1}} 1 / \psi(t)$ and hence

$$
\int_{J_{1}} \frac{d x}{\psi\left(B /\left(3 \gamma^{\prime}(\epsilon)\right)+x\right)^{2}} \leq D \int_{l_{l+1}} \frac{d x}{\psi\left(B /\left(3 \gamma^{\prime}(\epsilon)\right)+x\right)^{2}}
$$

This implies that

$$
\begin{aligned}
\int\left|T_{0} f_{\epsilon}(x)\right|^{2} d x \geq & \frac{C}{\gamma^{\prime}(\epsilon)^{2}}\left(\int_{I_{k^{\prime}}} \frac{d x}{\psi\left(B /\left(3 \gamma^{\prime}(\epsilon)\right)+x\right)^{2}}\right. \\
& \left.+\int_{\bigcup_{k=1}^{t^{\prime}-1}\left(I_{k} \cup J_{k}\right)} \frac{d x}{\psi\left(B /\left(3 \gamma^{\prime}(\epsilon)\right)+x\right)^{2}}\right) \\
\geq & \frac{C}{\gamma^{\prime}(\epsilon)^{2}} \int_{\epsilon}^{|\gamma|^{-1}(7)} \frac{d x}{\psi\left(B /\left(3 \gamma^{\prime}(\epsilon)\right)+x\right)^{2}} .
\end{aligned}
$$

For $\epsilon \leq \delta \leq|\gamma|^{-1}(7)$ we have

$$
\int\left|T_{0} f_{\epsilon}(x)\right|^{2} d x \geq \frac{C}{\gamma^{\prime}(\epsilon)^{2}} \int_{\epsilon}^{\delta} \frac{d x}{\psi\left(B /\left(3 \gamma^{\prime}(\epsilon)\right)+x\right)^{2}} .
$$

Since we are assuming that $T_{0}: L^{2}(\mathbb{R}) \rightarrow L^{2}(\mathbb{R})$ we have

$$
\int\left|T_{0} f_{\epsilon}\right|^{2}(x) d x \leq\left\|T_{0}\right\|_{L^{2} \rightarrow L^{2}}^{2}\left\|f_{\epsilon}\right\|_{2}^{2} \leq\left\|T_{0}\right\|_{L^{2} \rightarrow L^{2}}^{2} \frac{B}{3 \gamma^{\prime}(\epsilon)} .
$$

So finally we have

$$
\left\|T_{0}\right\|_{L^{2} \rightarrow L^{2}}^{2} \frac{B}{3 \gamma^{\prime}(\epsilon)} \geq \frac{C}{\gamma^{\prime}(\epsilon)^{2}} \int_{\epsilon}^{\delta} \frac{d x}{\psi\left(B /\left(3 \gamma^{\prime}(\epsilon)\right)+x\right)^{2}}
$$

or

(8)

$$
\begin{aligned}
1 & \geq \frac{C}{\gamma^{\prime}(\epsilon)} \int_{\epsilon}^{\delta} \frac{d x}{\psi\left(B /\left(3 \gamma^{\prime}(\epsilon)\right)+x\right)^{2}} \\
& \geq \frac{C}{\gamma^{\prime}(\epsilon)} \int_{\epsilon}^{\delta}-\gamma^{\prime \prime}\left(\frac{B}{3 \gamma^{\prime}(\epsilon)}+x\right) g\left(\frac{B}{3 \gamma^{\prime}(\epsilon)}+x\right) d x \\
& \geq \frac{C}{\gamma^{\prime}(\epsilon)} g\left(\frac{B}{3 \gamma^{\prime}(\epsilon)}+\delta\right) \int_{\epsilon}^{\delta}-\gamma^{\prime \prime}\left(\frac{B}{3 \gamma^{\prime}(\epsilon)}+x\right) d x \\
& =\frac{C}{\gamma^{\prime}(\epsilon)} g\left(\frac{B}{3 \gamma^{\prime}(\epsilon)}+\delta\right)\left[\gamma^{\prime}\left(\frac{B}{3 \gamma^{\prime}(\epsilon)}+\epsilon\right)-\gamma^{\prime}\left(\frac{B}{3 \gamma^{\prime}(\epsilon)}+\delta\right)\right] .
\end{aligned}
$$

Now, $g\left(B /\left(3 \gamma^{\prime}(\epsilon)\right)+\delta\right) \geq g(2 \delta)$ by also requiring $\delta>B /\left(3 \gamma^{\prime}(\epsilon)\right)$. 
The proof that

$$
\lim _{\epsilon \rightarrow 0^{+}} \frac{\gamma^{\prime}\left(\epsilon+1 / \gamma^{\prime}(\epsilon)\right)}{\gamma^{\prime}(\epsilon)}=1
$$

gives us that

$$
\lim _{\epsilon \rightarrow 0^{+}} \frac{\gamma^{\prime}\left(\epsilon+B /\left(3 \gamma^{\prime}(\epsilon)\right)\right)}{\gamma^{\prime}(\epsilon)}=1
$$

and hence we have that

$$
\frac{1}{\gamma^{\prime}(\epsilon)}\left[\gamma^{\prime}\left(\epsilon+\frac{B}{3 \gamma^{\prime}(\epsilon)}\right)-\gamma^{\prime}\left(\delta+\frac{B}{3 \gamma^{\prime}(\epsilon)}\right)\right] \rightarrow 1, \quad \text { as } \epsilon \rightarrow 0^{+} .
$$

So letting $\epsilon \rightarrow 0^{+}$in inequality (8) we get $1 \geq C g(2 \delta)$ and letting $\delta \rightarrow 0^{+}$we have a contradiction. This concludes the proof of Theorem 1.6.

Now let us prove Lemma 4.1 .

PROOF OF LEMMA 4.1. (i) Using the mean value theorem we have:

$$
|\gamma|^{-1}\left(2 k \pi-\frac{\pi}{3}\right)-|\gamma|^{-1}\left(2 k \pi+\frac{\pi}{3}\right)=\left(-\frac{2 \pi}{3}\right)\left(|\gamma|^{-1}\right)^{\prime}(d)=\frac{2 \pi}{3} \frac{1}{\gamma^{\prime}\left(|\gamma|^{-1}(d)\right)}
$$

for some $d$ such that $2 k \pi-\pi / 3 \leq d \leq 2 k \pi+\pi / 3$.

Since there is an $A \geq 1$ such that $\gamma^{\prime}\left(|\gamma|^{-1}(2 k)\right) \leq A \gamma^{\prime}\left(|\gamma|^{-1}(k)\right)$ and $\gamma^{\prime}\left(|\gamma|^{-1}(t)\right)$ is increasing we have that

$$
\begin{aligned}
\frac{1}{\gamma^{\prime}\left(|\gamma|^{-1}(d)\right)} & \geq \frac{1}{\gamma^{\prime}\left(|\gamma|^{-1}(2 k \pi+\pi / 3)\right.} \geq \frac{1}{\gamma^{\prime}\left(|\gamma|^{-1}(8 k)\right.} \\
& \geq A^{-3} \frac{1}{\gamma^{\prime}\left(|\gamma|^{-1}(k)\right)} \geq \frac{1}{A^{3} \gamma^{\prime}(\epsilon)} .
\end{aligned}
$$

Hence we have $|\gamma|^{-1}(2 k \pi-\pi / 3)-|\gamma|^{-1}(2 k \pi+\pi / 3) \geq 2 \pi /\left(3 A^{3} \gamma^{\prime}(\epsilon)\right)$.

(ii) It is enough to prove

(a) $\epsilon \geq|\gamma|^{-1}\left(2 k^{\prime} \pi+\pi / 3\right)+B /\left(3 \gamma^{\prime}(\epsilon)\right)$ and

(b) $|\gamma|^{-1}(7) \leq|\gamma|^{-1}(2 \pi-\pi / 3)-B /\left(3 \gamma^{\prime}(\epsilon)\right)$.

(a) Since $k^{\prime} \leq|\gamma|(\epsilon)<k^{\prime}+1 \leq 2 k^{\prime} \pi-\pi / 3$ we have that

$$
\begin{aligned}
\epsilon & \geq|\gamma|^{-1}\left(2 k^{\prime} \pi-\frac{\pi}{3}\right) \geq|\gamma|^{-1}\left(2 k^{\prime} \pi+\frac{\pi}{3}\right)+\frac{B}{\gamma^{\prime}(\epsilon)} \\
& \geq|\gamma|^{-1}\left(2 k^{\prime} \pi+\frac{\pi}{3}\right)+\frac{B}{3 \gamma^{\prime}(\epsilon)} .
\end{aligned}
$$


(b) Let

$$
B_{0}=\min \left[|\gamma|^{-1}(7), \gamma^{\prime-1}\left(\frac{B}{3\left(|\gamma|^{-1}(2 \pi-\pi / 3)-|\gamma|^{-1}(7)\right)}\right)\right] .
$$

Since $\epsilon \leq B_{0}$ we have

$$
\begin{aligned}
& \epsilon \leq \gamma^{\prime-1}\left(\frac{B}{3\left(|\gamma|^{-1}(2 \pi-\pi / 3)-|\gamma|^{-1}(7)\right)}\right) \\
& \Leftrightarrow \quad \gamma^{\prime}(\epsilon) \geq \frac{B}{3\left(|\gamma|^{-1}(2 \pi-\pi / 3)-|\gamma|^{-1}(7)\right)} \\
& \Leftrightarrow \quad \frac{B}{3 \gamma^{\prime}(\epsilon)} \leq|\gamma|^{-1}\left(2 \pi-\frac{\pi}{3}\right)-|\gamma|^{-1}(7) \\
& \Leftrightarrow \quad|\gamma|^{-1}(7) \leq|\gamma|^{-1}\left(2 \pi-\frac{\pi}{3}\right)-\frac{B}{3 \gamma^{\prime}(\epsilon)} .
\end{aligned}
$$

(iii) Let $\alpha=2 k \pi+\pi / 3$ and $\beta=2 \pi / 3$, so that $2(k+1) \pi+\pi / 3=\alpha+3 \beta$.

Since

$$
\left|I_{k+1}\right|+\left|J_{k}\right|=|\gamma|^{-1}\left(2 k \pi+\frac{\pi}{3}\right)-|\gamma|^{-1}\left(2(k+1) \pi+\frac{\pi}{3}\right)
$$

then

$$
\left|I_{k+1}\right|+\left|J_{k}\right|=(-3 \beta)\left(|\gamma|^{-1}\right)^{\prime}\left(t_{1}\right)=3 \beta \frac{1}{\gamma^{\prime}\left(|\gamma|^{-1}\left(t_{1}\right)\right)}
$$

for some $t_{1}$ such that $\alpha \leq t_{1} \leq \alpha+3 \beta$.

Also

$$
\begin{aligned}
\left|I_{k+1}\right|+\frac{2 B}{3 \gamma^{\prime}(\epsilon)} & =|\gamma|^{-1}\left(2(k+1) \pi-\frac{\pi}{3}\right)-|\gamma|^{-1}\left(2(k+1) \pi+\frac{\pi}{3}\right) \\
& =|\gamma|^{-1}(\alpha+2 \beta)-|\gamma|^{-1}(\alpha+3 \beta) \\
& =-\beta\left(|\gamma|^{-1}\right)^{\prime}\left(t_{2}\right) \\
& =\beta \frac{1}{\gamma^{\prime}\left(|\gamma|^{-1}\left(t_{2}\right)\right)}
\end{aligned}
$$

for some $t_{2}$ with $\alpha+2 \beta \leq t_{2} \leq \alpha+3 \beta$.

Since $t_{2} \leq \alpha+3 \beta \leq 2 \alpha \leq 2 t_{1}$, we have $t_{2} / 2 \leq t_{1}$. Using the doubling property of $\gamma^{\prime}\left(|\gamma|^{-1}(t)\right)$ we get $\gamma^{\prime}\left(|\gamma|^{-1}\left(t_{2} / 2\right)\right) \geq \frac{1}{A} \gamma^{\prime}\left(|\gamma|^{-1}\left(t_{2}\right)\right)$.

So finally we see that

$$
\begin{aligned}
\left|I_{k+1}\right|+\left|J_{k}\right| & =3 \beta \frac{1}{\gamma^{\prime}\left(|\gamma|^{-1}\left(t_{1}\right)\right)} \leq 3 \beta \frac{1}{\gamma^{\prime}\left(|\gamma|^{-1}\left(t_{2} / 2\right)\right)} \\
& \leq 3 \beta A \frac{1}{\gamma^{\prime}\left(|\gamma|^{-1}\left(t_{2}\right)\right)}=3 A\left(\left|I_{k+1}\right|+\frac{2 B}{3 \gamma^{\prime}(\epsilon)}\right) \\
& \leq 3 A\left(\left|I_{k+1}\right|+2\left|I_{k+1}\right|\right) .
\end{aligned}
$$

So $\left|J_{k}\right| \leq(9 A-1)\left|I_{k+1}\right|$. This ends the proof of Lemma 4.1. 


\section{Acknowledgement}

I would like to thank James Wright for his most valuable comments.

\section{References}

[1] C. Fefferman and E. M. Stein, ' $H^{p}$-spaces of several variables', Acta Math 129 (1972), 137-193.

[2] I. I. Hirschman, 'On multiplier transformations', Duke Math. J. 26 (1959), 221-242.

[3] B. H. Shayya, A class of strongly singular integral operators (Ph.D. Thesis, University of Wisconsin, 1996).

[4] E. M. Stein, Harmonic analysis: real-variable methods, orthogonality, and oscillatory integrals (Princeton Univ. Press, Princeton NJ, 1993).

[5] E. M. Stein and G. Weiss, 'On the interpolation of analytic families of operators acting on $H^{p}$. spaces', Tôhoku Math. J. 9 (1957), 318-339.

Instituto de Matemáticas

Universidad Nacional Autónoma de México

Circuito Exterior, Cd. Universitaria

México D.F. 04510

México

e-mail: folchgab@matem.unam.mx 